\title{
Incidental diagnosis of extra ovarian primary peritoneal carcinoma at caesarean section
}

\author{
Delphine Rose*, Soundararajan Pradeeba
}

Department of Obstetrics and Gynaecology, Thanjavur Medical College, Thanjavur, Tamil Nadu, India

Received: 28 July 2016

Accepted: 28 August 2016

\section{*Correspondence:}

Dr. Delphine Rose,

E-mail: drdelphinerose@gmail.com

Copyright: () the author(s), publisher and licensee Medip Academy. This is an open-access article distributed under the terms of the Creative Commons Attribution Non-Commercial License, which permits unrestricted non-commercial use, distribution, and reproduction in any medium, provided the original work is properly cited.

\begin{abstract}
Extra ovarian primary peritoneal carcinoma is rare and interesting tumour characterized by peritoneal carcinomatosis with ascites and by a histological pattern similar to ovarian serous papillary adenocarcinoma. It is a relatively newly defined disease. Most reported cases of EOPPC have been in elderly women. Here we report a case of EOPPC diagnosed incidentally while performing an emergency caesarean section in an otherwise healthy and young primigravida. Patient had diffuse metastatic peritoneal deposits peroperatively. She was started on chemotherapy.
\end{abstract}

Keywords: Peritoneal carcinoma, Caesarean section, Metastasis

\section{INTRODUCTION}

Extra ovarian primary peritoneal carcinoma (EOPPC) is a rare malignant epithelial tumour that develops from the peritoneum lining the pelvis and abdomen and is characterized by abdominal carcinomatosis, uninvolved or minimally involved ovaries, and no identifiable primary tumor. ${ }^{1}$ It is similar to serous ovarian carcinoma with respect to clinical presentation, histological appearance, pattern of spread, treatment, and prognosis. ${ }^{2}$ This entity has been reported under various names including serous surface papillary carcinoma of the peritoneum, extra ovarian mullerian adenocarcinoma, multiple focal extra ovarian serous carcinoma and normal sized ovary carcinoma syndrome. ${ }^{2,3}$

\section{CASE REPORT}

A 27 year old Mrs. X, a primigravida at term gestation was admitted in our labour ward with labour pains and leaking per vaginum for 6hrs. There were no significant medical or surgical illness in the past. She attained menarche at the age of 12 and had regular menstrual cycles with normal flow. She was married for 2 years and had conceived spontaneously. No positive family and personal history. All three trimesters were uneventful. Her appetite was good and weight gain was $9 \mathrm{~kg}$. Her antenatal ultrasound revealed normal growth of the foetus.

On examination, the general condition of the patient was good, there was no pallor or pedal oedema, temperature was normal and her vitals were stable. On abdominal examination, uterus was term, with a cephalic presentation and a good foetal heart. Per vaginum, cervix was soft, central, $50 \%$ effaced, OS $2 \mathrm{~cm}$. dilated, absent membranes, head at -2 station, a gynaecoid pelvis with clear liquor draining. Baseline investigations were normal. CTG was reactive. Labour was augmented with 5 units oxytocin infusion. After $12 \mathrm{hrs}$ patient were taken up for emergency caesarean section for non-progress of labour and an alive term female baby of $2.8 \mathrm{~kg}$ was delivered with a good Apgar.

Peroperatively, there was around $100 \mathrm{ml}$ of straw coloured peritoneal fluid. There were multiple nodular deposits over the peritoneum, anterior and posterior surfaces of uterus, fallopian tube, ovary and pouch of Douglas. Both 
ovaries were enlarged and cystic. Other organs were found to be normal. Multiple biopsies from the deposits over the peritoneum, uterus and ovaries were taken and abdomen closed.

Patient was evaluated in the post-operative period.CA 125 was $12089 \mathrm{u} / \mathrm{ml}$. CECT abdomen and pelvis showed cystic lesion with thin internal septations in both ovaries, nodules within the peritoneal cavity and POD with free fluid. OGD Scopy and Colonoscopy were normal. Histopathological examination showed papillary serous adenocarcinoma with psammoma bodies. No primary site was found anywhere. Hence a diagnosis of EOPPC was made. Patient was started on chemotherapy and currently under third cycle of drugs.

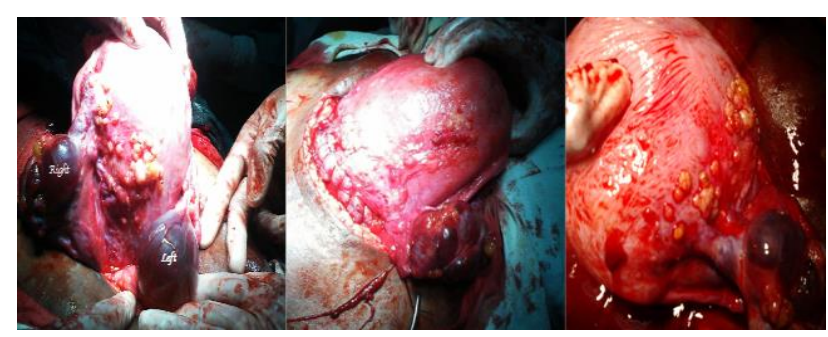

Figure 1: Metastatic deposits over the uterus with enlarged ovaries as seen during caesarean section.

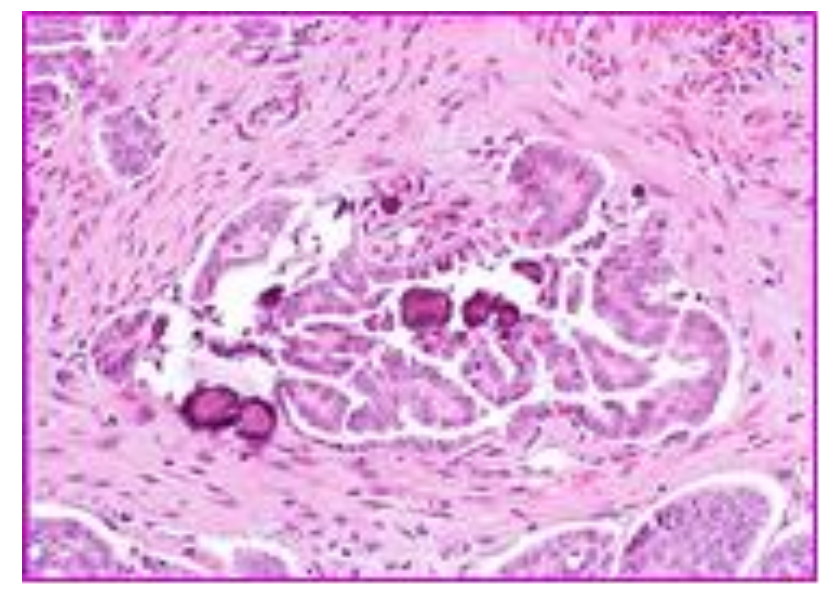

Figure 2: Histopathological examination showed deposits of serous papillary adenocarcinoma with psammoma bodies.

\section{DISCUSSION}

EOPPC is a rare adenocarcinoma that arises in the peritoneum. The age distribution and clinical presentation is indistinguishable from that of advanced-stage epithelial ovarian cancer. ${ }^{5}$ Most reported cases of EOPPC have been in elderly women. Insert sentence - However, rare cases have been reported in children and males. ${ }^{6,7}$ Patients ordinarily present with non-specific abdominal symptoms and ascites reported in approximately $85 \%$ of cases. ${ }^{1}$ At laparotomy, almost all cases show diffuse peritoneal tumour implants, which usually involve the omentum and upper abdomen. Although EOPPC always involves the full thickness of the omentum, invasion into other abdominal or pelvic organs is rare and, when present, tends to be superficial. The ovaries are almost always of normal size and shape, and frequently display surface tumor implants which may be focally invasive; rarely, they may be normal grossly and microscopically. ${ }^{2}$ These findings are similar to those of advanced-stage epithelial ovarian cancer or peritoneal carcinomatosis from metastatic gastrointestinal cancers, except that the ovaries show minimal or no involvement and no primary can be found in the gastrointestinal tract or other organs. ${ }^{1}$ Identification of the correct primary site is critical because the surgical management of EOPPC is vastly different from that of carcinomatosis associated with other malignancies. ${ }^{5}$ Although EOPPC may be considered in the differential diagnosis, it is not a diagnosis that can made preoperatively. The diagnosis of EOPPC is typically made by exclusion after both operative assessment and pathological study. If ovaries seem normal with widespread disease elsewhere in the abdomen, EOPPC becomes a leading diagnostic possibility. However, because surface involvement of the ovaries is present in approximately $96 \%$ of the cases, the distinction between EOPPC and epithelial ovarian carcinoma may only be made after histological examination to evaluate the extent of ovarian invasion by tumour. $^{5}$

EOPPC spreads mainly transperitonealy; however, lymphatic and blood-borne metastases have been suggested. Metastases to different groups of lymph nodes, the liver, and the brain have been reported. ${ }^{8,9}$ Most cases of EOPPC reported in the literature have been of serous histology. However, other histologic variants of the müllerian system have been reported; specifically, endometrioid, clear cell, mucinous, Brenner tumor, and mixed müllerian tumors; but nonserous and serous tumors appear to be similar with regard to prognosis and response to therapy. ${ }^{10}$

The light microscopic, histochemical, immunohistochemical and ultrastructural features of EOPPC are similar to those of ovarian serous carcinoma. Thus, EOPPC appears as a high-grade, purely epithelial neoplasm with frequent mitotic figures, necrosis, slitlike glandular spaces, and psammoma bodies. ${ }^{11}$

In order to differentiate EOPPC from papillary serous adenocarcinoma of the ovary, the Gynecologic Oncology Group has stipulated that the following criteria be met: ${ }^{2}$

1. Histology must be predominantly serous or identical to any grade of ovarian papillary serous tumor.

2. The ovaries are of normal size or enlarged by a benign process.

3. The involvement in the extra ovarian sites must be greater than the involvement on the surface of either ovary and 
4. The ovarian component must be nonexistent or confined to surface epithelium or less than $5 \times 5 \mathrm{~mm}$. within the stroma.

Furthermore, some authors suggested that genetic events (HER-2/neu overexpression) responsible for malignant transformation in EOPPC may be distinct from those responsible for epithelial ovarian cancer. ${ }^{11}$ Also, EOPPC must be differentiated from malignant mesothelioma, benign papillary mesothelioma, metastatic peritoneal carcinomatosis, borderline primary peritoneal serous tumor, endosalpingiosis, and psammocarcinoma of the peritoneum. Malignant mesothelioma is closely related to long-term exposure to asbestos, has a male predominance, frequent spindle cell component, cytoplasmic eosinophilia, and sometimes extensive cell vacuolization, and rare psammoma bodies. Previous studies have shown that the expression of B72.3, PLAP, or CEA by a papillary peritoneal tumor would militate against a diagnosis of mesothelioma; also combined reactivity for S-100 and PLAP, or S-100 and B72.3, characterizes the majority of serous adenocarcinomas, and is not observed in mesotheliomas. ${ }^{12}$ Ordoñez reported that from the practical point of view, calretinin, thrombomodulin, and keratin $5 / 6$ are the best positive markers for distinguishing between epithelial malignant mesotheliomas and papillary serous carcinomas diffusely involving the peritoneum. Among the antibodies that are considered to be negative markers for mesothelioma, MOC-31, B72.3, Ber-EP4, CA19-9, and Leu-M1 proved to the best diagnostic discriminators. ${ }^{13}$ Other investigators have found that a two-marker panel of antibodies including vimentin and Ber-EP4 is most useful for the differential diagnosis between carcinoma and mesothelioma. ${ }^{14}$ Also, recently, Ordoñez indicate that because ER is frequently expressed in serous carcinomas but not in mesotheliomas, this marker could be very useful to discriminate between these malignancies. ${ }^{15}$ Benign papillary mesothelioma occurs in both men and women, usually of young age, has well-formed papillae, mostly lined by one layer of a single cell type that resemble reactive mesothelium, and showing little or no anaplasia or mitoses; and an absence of invasion into the peritoneum or abdominopelvic organs. ${ }^{16}$ To the best of our knowledge, no morphologic features can afford a reliable distinction between EOPPC and metastatic peritoneal carcinomatosis; diagnosis of the latter rests on recognizing a primary tumor, usually in the ovary, fallopian tube, or endometrium and less frequently in other organs such as breast, gastrointestinal tract (especially stomach, pancreas), lungs and thyroid gland. ${ }^{11}$ Primary serous borderline tumors of the peritoneum have also been reported, albeit in fewer numbers than carcinomas, affecting younger patients, and having the microscopic features of ovarian borderline serous tumor. These primary serous borderline tumors have an excellent prognosis, although rare cases have been reported in which transformation to carcinoma has been observed on follow-up examination. ${ }^{17}$ Endosalpingiosis is a benign lesion, found most often in association with chronic salpingitis; it is most commonly encountered in the pelvic peritoneum but rarely involves other portions of the peritoneum, and consists of glandular inclusions lined by normal-appearing tubal-type epithelium. ${ }^{18}$ Also, another less virulent variation of EOPPC is discussed in the literature. ${ }^{19}$ This is the serous psammocarcinoma of the peritoneum, has a proportionately larger number of psammoma bodies and a less-aggressive cytologic appearance with absent or, at most, moderate nuclear atypia and rare mitotic figures. ${ }^{20}$

The pathogenesis of EOPPC has been controversial. Some authors believe that embryonic germ cell rests remain along the gonadal embryonic pathway and that EOPPC develops from a malignant transformation of these cells. ${ }^{12}$ Other authors contend that field carcinogenesis occurs, with the celomic epithelium lining the abdominal cavity (peritoneum) and the ovaries (germinal epithelium) manifesting a common response to an oncogenic stimulus. ${ }^{18}$ Muto et al. have suggested a multifocal origin with clonality studies. ${ }^{21}$ However, Kupryjanczyk et al. have identified others findings that are consistent with a unifocal origin. ${ }^{22}$ Therefore, more extensive studies of this type should be performed to confirm these results. Recently, Euscher et al investigated the WT-1 expression in serous carcinoma arising from different sites within the female genital tract and suggested that serous carcinoma may have a different biology based on site of origin. ${ }^{23}$

The prognosis of EOPPC is poor. Medial survival time vary between 7 and 27.8 months, while 5-year survival rates range from $0 \%$ to $26.5 \%^{8}$. Treatment usually includes abdominal hysterectomy, bilateral salpingooophorectomy, and tumour debulking followed by chemotherapy. Surgery remains critically important for both the diagnosis and the therapy of EOPPC. Once the diagnosis has been established and the extent of disease documented, maximal cytoreduction becomes the primary goal of the procedure. Excision of all visible implants is the hallmark of cytoreductive efforts. The subsequent courses include, in most cases, initial good response followed months later by uncontrollable, lethal local recurrence; rapid and lethal progression of local disease without any evidence of initial response to chemotherapy; and, in rare case, long-term maintenance of the initial response, with the patient being considered cured. ${ }^{5}$

\section{CONCLUSION}

EOPPC is a relatively newly defined disease that accounts for approximately $10 \%$ of cases with a presumed diagnosis of ovarian cancer. It is characterized by abdominal carcinomatosis, uninvolved or minimally involved ovaries and no identifiable primary. It is similar in clinical presentation, histologic appearance and response to serous ovarian carcinoma. The median survival rate is less than 2 years in most cases. 
Cytoreduction followed by cisplatin based multiagent therapy is the mainstay of treatment.

Informed consent was obtained from the patient.

Funding: No funding sources

Conflict of interest: None declared

Ethical approval: Not required

\section{REFERENCES}

1. Eltabbakh GH, Piver MS. Extraovarian primary peritoneal carcinoma. Oncology (Willinston Park). 1998;12:813-25.

2. Bloss JD, Liao SY, Buller RE, Manett A, Berman ML, McMeekin S, et al. Extraovarian peritoneal serous papillary carcinoma: a case-control retrospective comparison to papillary adenocarcinoma of the ovary. Gynecol Oncol. 1993;50:347-51.

3. Feuer GA, Shevchuk M, Calanog A. Normal-sized ovary carcinoma syndrome. Obstet Gynecol. 1989;73:786-92.

4. Swerdlow M. Mesothelioma of the pelvic peritoneum resembling papillary cystadenocarcinoma of the ovary. Am J Obstet Gynecol. 1959;77:197-200.

5. Chu Cs, Menzin AW, Leonard DGB, Rubin SC, Wheeler JE. Primary peritoneal carcinoma: A review of the literature. Obstet Gynecol Surv. 1999;54:32335.

6. Shibata R, Matsufuji H, Morimoto T, Hosoya R, Araki K, Hata J. Extraovarian primary peritoneal carcinoma in a child. Pediatr Blood Cancer. 2004;42:292-3.

7. Shmueli E, Leider-Trejo L, Schwartz I, Aderka D, Inbar M. Primary papillary serous carcinoma of the peritoneum in a man. Ann Oncol. 2001;12:563-7.

8. Fromm GL, Gershenson DM, Silva EG. Papillary serous carcinoma of the peritoneum. Obstet Gynecol. 1990;75:75-89.

9. Eltabbakh GH, Piver MS, Werness BA. Primary peritoneal adenocarcinoma metastatic to the brain. Gynecol Oncol. 1997;66:160-3.

10. Altaras MM, Aviram R, Cohen I, Cordoba M, Weiss E, Beyth Y. Primary peritoneal papillary serous adenocarcinoma: clinical and management aspect. Gynecol Oncol. 1991;40:230-6.
11. Mills SE, Andersen WA, Fechner RE, Austin MB. Serous surface papillary carcinoma. A clinicopathologic study of 10 cases and comparison with stage III-IV ovarian serous carcinoma. Am J Surg Pathol. 1998;12:827-34.

12. Bollinger DJ, Wick MR, Dehner LP, Mills SE, Swanson PE, Clarke RE. Peritoneal malignant mesothelioma versus serous papillary adenocarcinoma. Am J Surg Pathol. 1989;13:659-70.

13. Ordoñez NG. Role of immunohistochemistry in distinguishing epithelial peritoneal mesotheliomas from peritoneal and ovarian serous carcinomas. Am J Surg Pathol. 1998;22:1203-14.

14. García-Prats MD, Ballestin C, Sotelo T, LopezEncuentra A, Mayordomo JI. A comparative evaluation of immunohistochemical markers for the differential diagnosis of malignant pleural tumours. Histopathology. 1998;32:462-72.

15. Ordoñez NG. Value of estrogen and progesterone receptor immunostaining in distinguishing between peritoneal mesotheliomas and serous carcinomas. Hum Pathol 2005; 36:1163-1167.

16. Goepel JR. Benign papillary mesothelioma of peritoneum: A histological, histochemical and ultrastructural study of six cases. Histopathology. 1987;5:21-30.

17. Bell DA, Scully RE. Serous borderline tumors of the peritoneum. Am J Surg Pathol. 1990;14:230-49.

18. Zinser KR, Wheeler JE. Endosalpingiosis in the omentum. A study of autopsy and surgical material. Am J Surg Pathol. 1982;6:109-17.

19. Gilks CB, Bell DA, Scully RE. Serous psammocarcinoma of the ovary and peritoneum. Int $\mathbf{J}$ Gynecol Pathol. 1990;9:110-21.

20. Kannerstein M, Churg J. Peritoneal mesothelioma. Hum Pathol. 1997;8: 83-94.

21. Muto MG, Welh WR, Mok SC, Bandera CA, Fishbaugh PM, Tsao SW, et al. Evidence for a multifocal origin of papillary serous carcinoma of the peritoneum. Cancer Res.1995;55:490-2.

22. Kupryjanczyk J, Thor AD, Beauchamp R, Poremba C, Scully RE, Yandell DW. Ovarian, peritoneal, and endometrial serous carcinoma: Clonal origin of multifocal disease. Mod Pathol 1996; 9: 166-173.

23. Euscher ED, Malpica A, Deavers MT, Silva EG. Differential expression of WT-1 in serous carcinomas in the peritoneum with or without associated serous carcinoma in endometrial polyps. Am J Surg Pathol. 2005;29:1074-8.

Cite this article as: Rose D, Pradeeba S. Incidental diagnosis of extra ovarian primary peritoneal carcinoma at caesarean section. Int J Reprod Contracept Obstet Gynecol 2016;5:3597-600. 